\title{
Investigation of the Effect of Sulfur Heteroatom on Asphaltene Aggregation
}

\author{
Ana C. R. Soderoł:, Hugo Santos Silvat, Patricia Guevara Level:t, Brice Bouyssieref, Jean-Pierre \\ Korb§, Herve Carrier $\perp$, Isabelle Barailleł and Didier Béguét. \\ $\dagger$ Laboratório de Modelagem Molecular e QSAR (MODMOLQSAR), Departamento de Fármacos e \\ Medicamentos, Universidade Federal do Rio de Janeiro (UFRJ), Av. Carlos Chagas Filho, 373, 21941- \\ 599, Brazil. \\ $\ddagger$ Institut Pluridisciplinaire de Recherche sur l'Environnement et les Matériaux (IPREM), UMR 5254, \\ Université de Pau et des Pays de l'Adour, Hélioparc, 2 Avenue du Président Angot, 64053 Pau Cedex \\ 9, France.

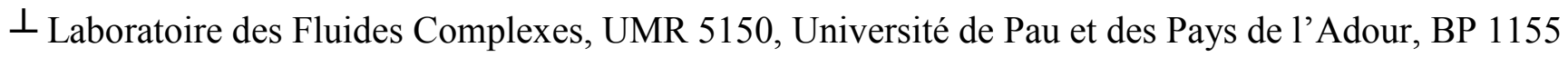 \\ 64013 Pau Cedex, France \\ $\S$ Physique de la Matière Condensée, Ecole Polytechnique-Centre National de la Recherche \\ Scientifique (CNRS), 91128 Palaiseau, France.
}

In order to study all possible positions of the sulfur atom at asphaltene molecules, we also performed the molecular dynamic simulation of molecules 5 and 6 (Figure S1 and Table S1), as dimers (systems $5 \mathrm{D}$ and $6 \mathrm{D}$ ) and trimers (systems 5T and 6T).

Figure S2 shows the initial (0 ns) and the final configurations (150 ns) of the four systems. Visual inspection of molecular dynamics simulation (MDS) trajectories indicates that systems $5 \mathrm{D}, 6 \mathrm{D}, 5 \mathrm{~T}$, and 5T are not stable during the molecular dynamics simulation in toluene and this regardless of the starting configuration. However, the systems presented different behavior.

For the "organized" starting configuration: the monomer from system 5D rotates at the beginning of simulation ( 1ns) and becomes separated during the MDS. At system 5T, one monomer rotates at 50 ns followed by side chains interaction. However, this configuration is not stable, since the monomers dissociated after $90 \mathrm{~ns}$. Systems 6D and 6T assume many orientations and remains aggregated during all simulation. Nevertheless, they do not adopt defined geometries. This behavior is typical of an interaction done exclusively by means of the acid chain end via hydrogen bonding.

Dimers (Systems 5D and 6D): System 5D shows variable distances after 30 ns (Figure S3). The molecules are close to each other $(<0.4 \mathrm{~nm})$ at only $15.4 \%(23 \mathrm{~ns})$ of the simulation (Table S2). System 6D shows instability since the beginning of simulation, with distances higher than $0.4 \mathrm{~nm}$ during the simulation time.

In order to investigate the mean type of interactions between monomers, the intermolecular contacts were analyzed. Figure $\mathrm{S} 5$ shows the numbers of $\pi-\pi, \pi-\theta$, and $\theta-\theta$ contacts as a function of simulation 
time for systems $5 \mathrm{D}$ and $6 \mathrm{D}$. At system $5 \mathrm{D}$, the average number of $\pi-\pi$ contacts rapidly decreases after $50 \mathrm{~ns}$, which demonstrate the molecules dissociation. The average number of $\pi-\pi$ contacts is 16 from 50 to $100 \mathrm{~ns}$, compared with 636 at system 1D. It can also elucidate the differences in the $\pi-\theta$ contacts (Table S3). The average number of $\pi-\theta$ contacts is 141 for system 1D, while is 35 for system 5D. Additionally, this divergence is indicated by the presence of hydrogen bonds between the carboxylic acid of side chains and the carbonyl group of poly-aromatic cores from system 1D. The side chains of system 1D interacts with the carbonyl group of poly-aromatic core during $54 \%$ (81 ns) of total simulation, but they do not interact through hydrogen bonds with each other. For system 6D, the number of $\pi-\pi$ contacts rapidly decreases after $5 \mathrm{~ns}$, due to monomers dissociation. However, like system $5 \mathrm{D}$, the number of $\theta-\theta$ contacts does not suffer great changesand it occurs during all the simulations.

The cosine of angle between aromatic cores was also calculated to describe the geometry of asphaltene molecules and the most favorable arrangement in asphaltene aggregation. ${ }^{1}$ Values of $\omega=0^{\circ}$ $(\cos \omega=1)$ or $\omega=180^{\circ}(\cos \omega=-1)$ corresponds to parallel monomers (polyaromatic cores as $\pi-\pi$ stacking), and $\omega=90^{\circ}$ ( $\cos \omega=0$ ) corresponds to perpendicular molecules (T-shape). The cosine value of 1 is present only at 20\% (30 ns) of total simulation for system $5 \mathrm{D}$ and $3 \%$ (4.5 ns) for system 6D, which suggests the molecules do not remain in a parallel geometry during the simulation. The geometry shows enormous variation during MDS, for both systems. Nevertheless, the parallel arrangement corresponds only to $14 \%$ (21 ns) of simulation at short distances between the monomers, to system 5D. This behavior was expected, since the molecules dissociate after $51 \mathrm{~ns}$.

Trimers (Systems 5T and 6T): The distances between monomers of systems indicate greater variation for system 5T and 6T (Figure S3 and Table S4). System 5T shows larger distances after 30 ns and the molecules are close to each other $(<0.4 \mathrm{~nm})$ at only $13 \%(19.5 \mathrm{~ns})$ of the simulation. System 5T shows larger distances during the entire simulation, due to monomers dissociation.

When the predominant type of interactions was investigated (Figure S4 and Table S3), it was noticed the average number of $\pi-\pi$ contacts is 957 for system $5 \mathrm{~T}$, although this number decreases to 650 from 90 to $150 \mathrm{~ns}$. The simulation results indicate the monomer detach from the trimer after $90 \mathrm{~ns}$, reducing the contacts between the poly-aromatic cores. The same behavior is observed to $\pi-\theta$ contacts. The average number of $\pi-\theta$ contacts is 172 , although it decreases to 109 from 90 to $150 \mathrm{~ns}$.

The number of contacts is very low to system 6T, since the monomers become apart from the beginning of simulation. The average number of $\pi-\pi, \pi-\theta$, and $\theta-\theta$ contacts is 48,38 , and 70 respectively. The change/deletion of the carbonyl/thionyl at the core has a great impact on $\pi-\pi$ and $\pi-\theta$ contacts, and it can significantly alter the aggregations formation since the systems show different behaviors.

The trimers geometry was also analyzed by the cosine values of the angle between poly-aromatic cores (Figure S5). For both systems, the cosine does experience large variation (Figure S5) and does not have association to short distances.

The side chains from trimers interact through hydrogen bonds during the simulation. At systems $5 \mathrm{~T}$, the frequency of hydrogen bonds between carboxylic groups of side chains is higher than 50\% (75 ns) of total simulation, but for system 6T, this number decreases to 26\% (39 ns). Moreover, the presence of carboxylic acid group is important to aggregation, but they were not enough to maintain the aggregated in a parallel orientation. 
Finally, in order to understand these results, the molecule 5 was studied by Density Functional Theory (DFT) using the B3LYP/D3BJ level of theory and def-TZVPP basis-set within the Orca 3.0.3 package. We then compared the molecular orbitals of molecule 5 with the ones of molecule 1 (in the main body of the article).

In Figure $\mathrm{S} 6$ one can find the $\mathrm{HOMO}$ orbitals of molecules 1 and 5 . The first one has $4 \mathrm{C}=\mathrm{O}$ bonds whereas the second one has $4 \mathrm{C}=\mathrm{S}$ bonds. One should note that the HOMO's electronic density is similar for the two molecules, except the contribution of the Sulfur atoms. In the case of molecule 5, this contribution is very important, what is seen by the increase of the density of the lobe located over these atoms. This should somehow avoid the $\pi$-stacking as demonstrated by molecular dynamics as well.

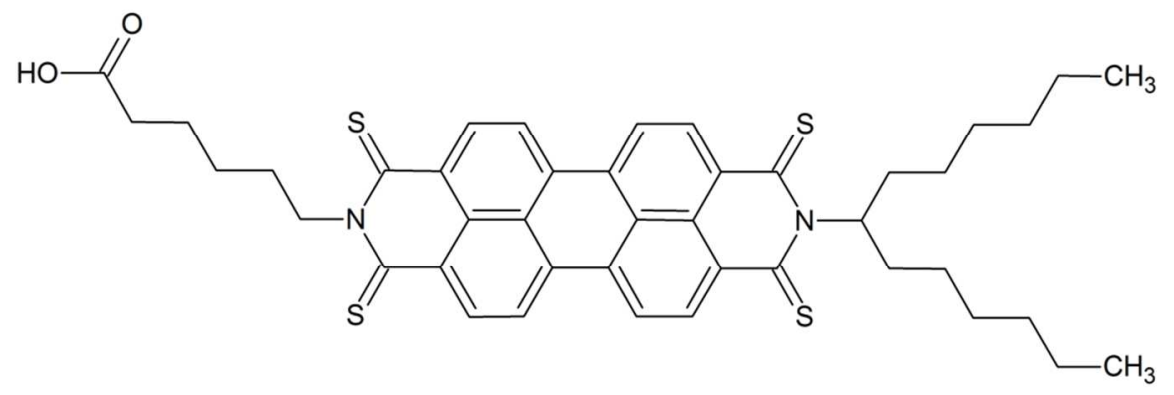

5

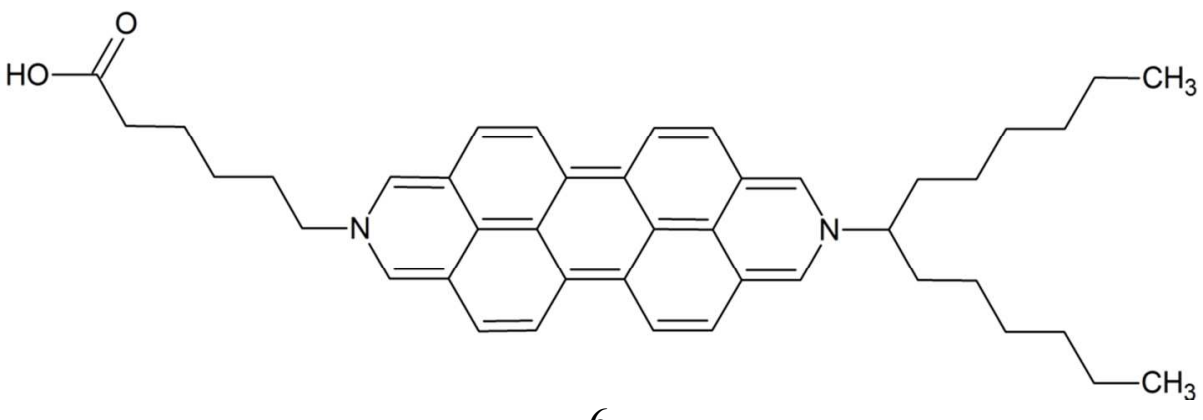

6

Figure S1. Molecular structures of asphaltene molecules used in this study. 

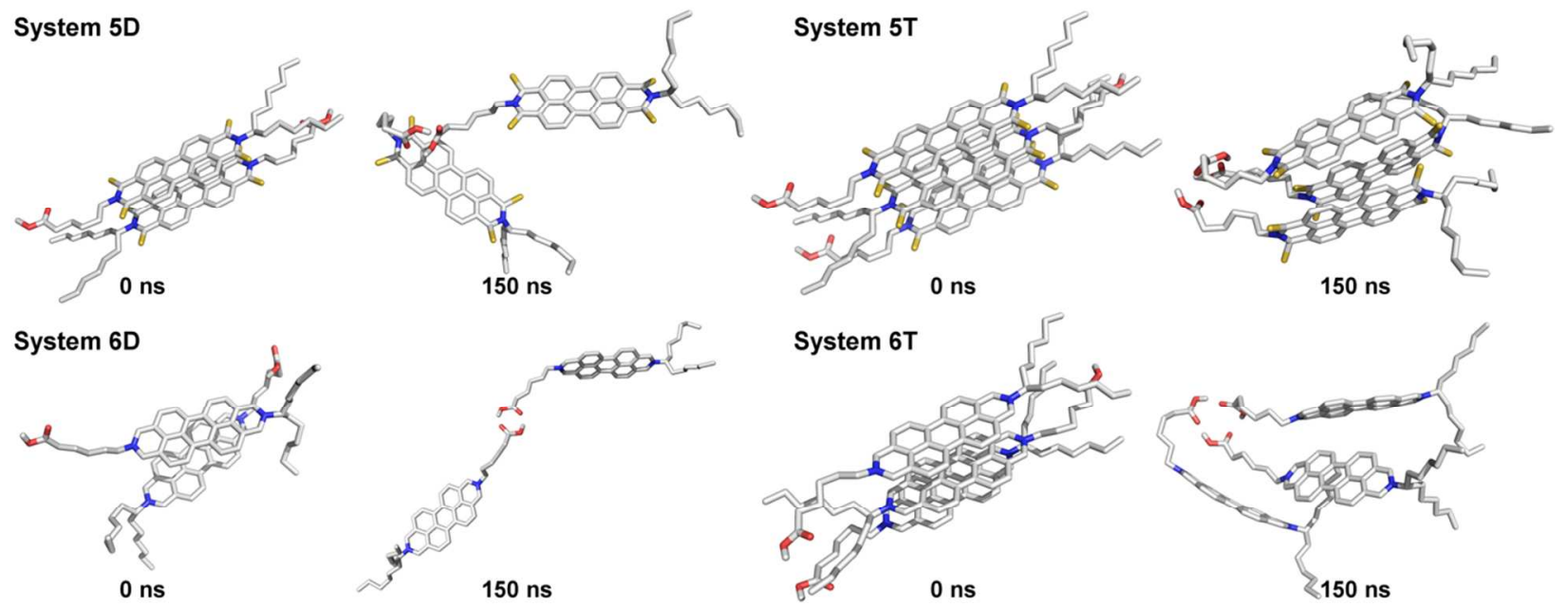

Figure S2. Snapshots of the initial configuration and the final frame of the trimers simulations. Carbon atoms are in grey, oxygen in red, nitrogen in blue and sulfur in yellow. Hydrogen atoms are omitted for clarity. 

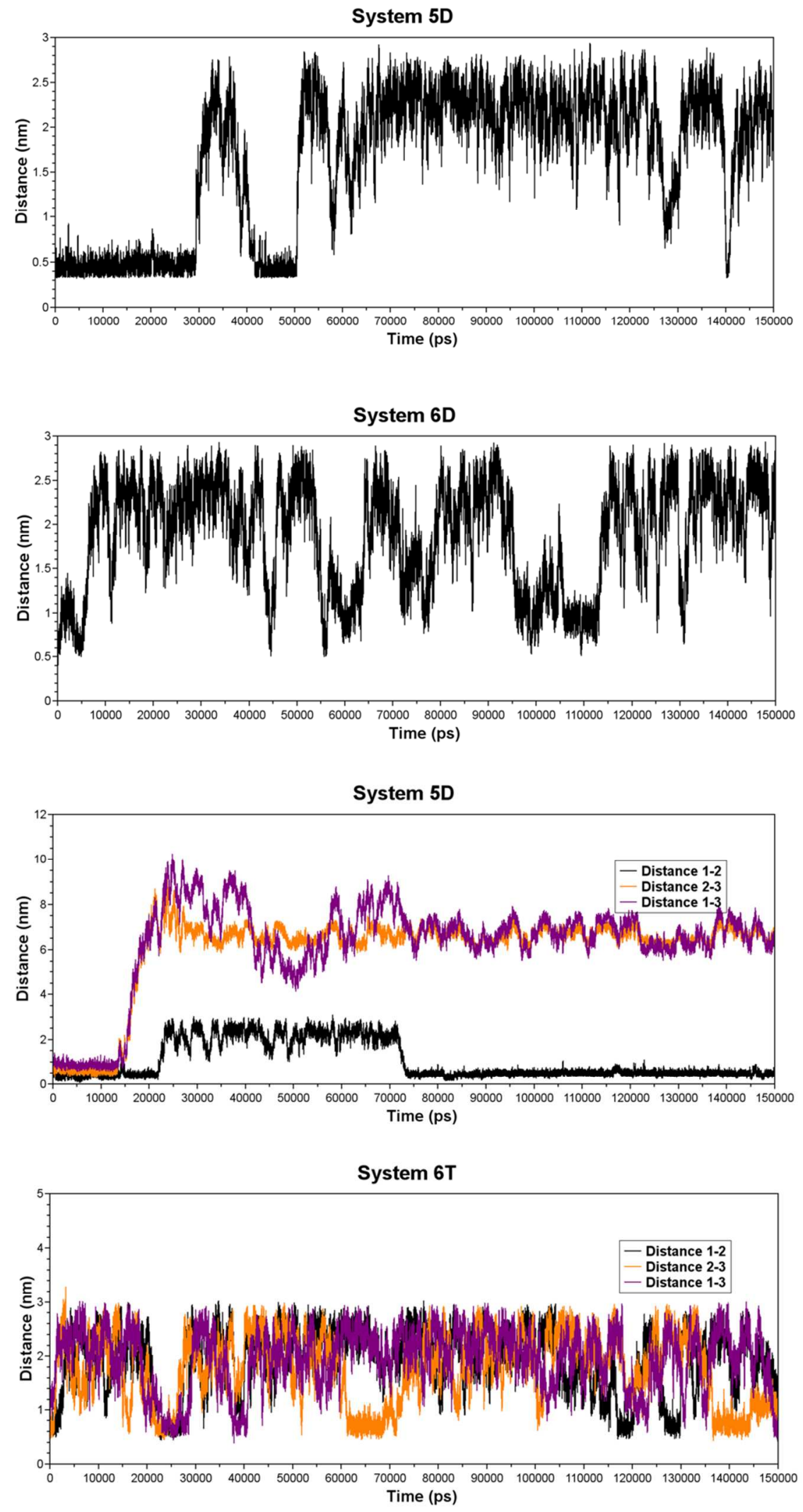

Figure S3. Distances between monomers of systems 5D, 6D, 5T, and 6T. 

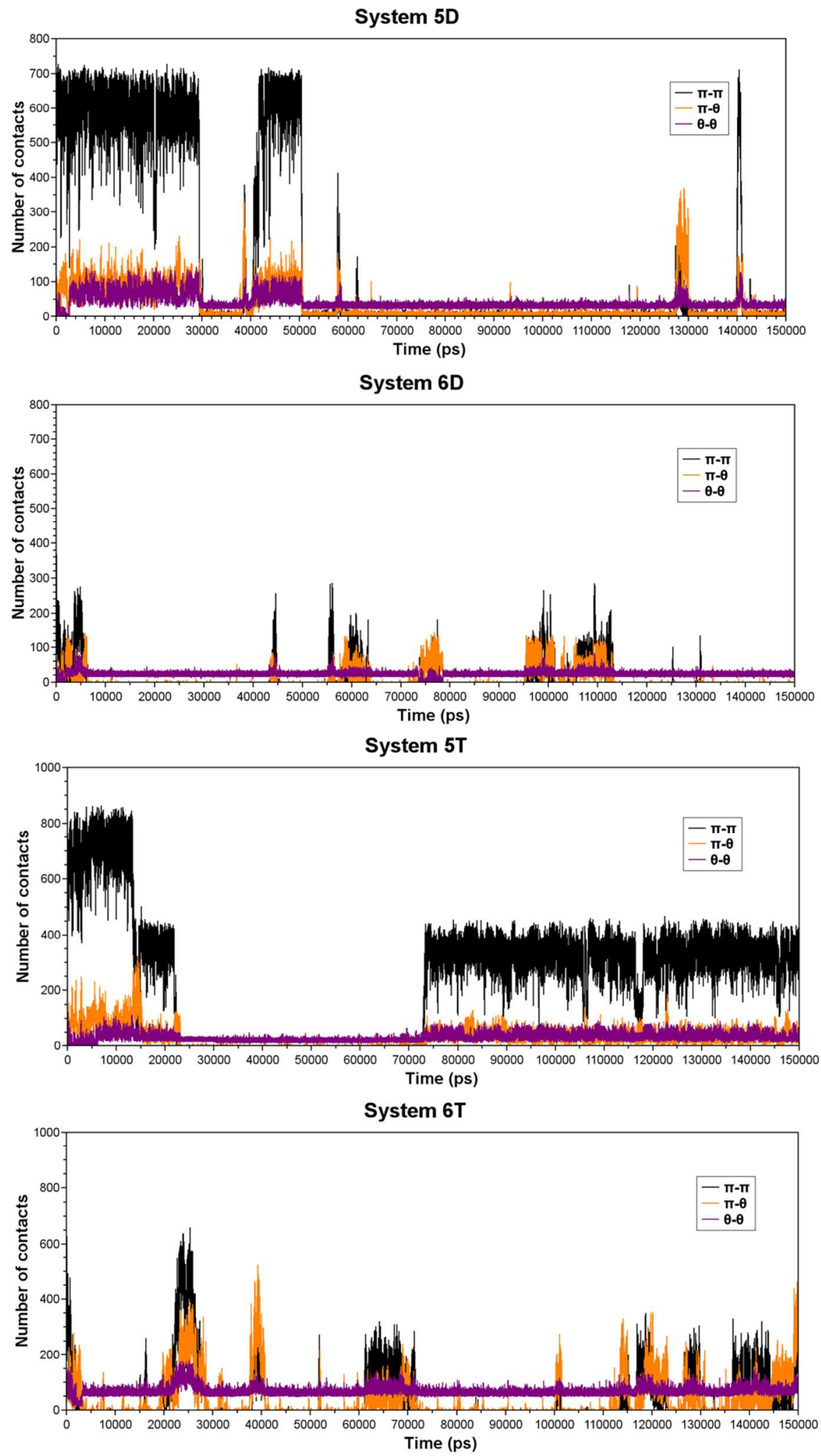

Figure S4. Number of contacts of systems $5 \mathrm{D}, 6 \mathrm{D}, 5 \mathrm{~T}$, and $6 \mathrm{~T}$. $\pi-\pi$ contacts are in black, $\theta-\pi$ contacts are in orange, and $\theta-\theta$ contacts are in purple. 

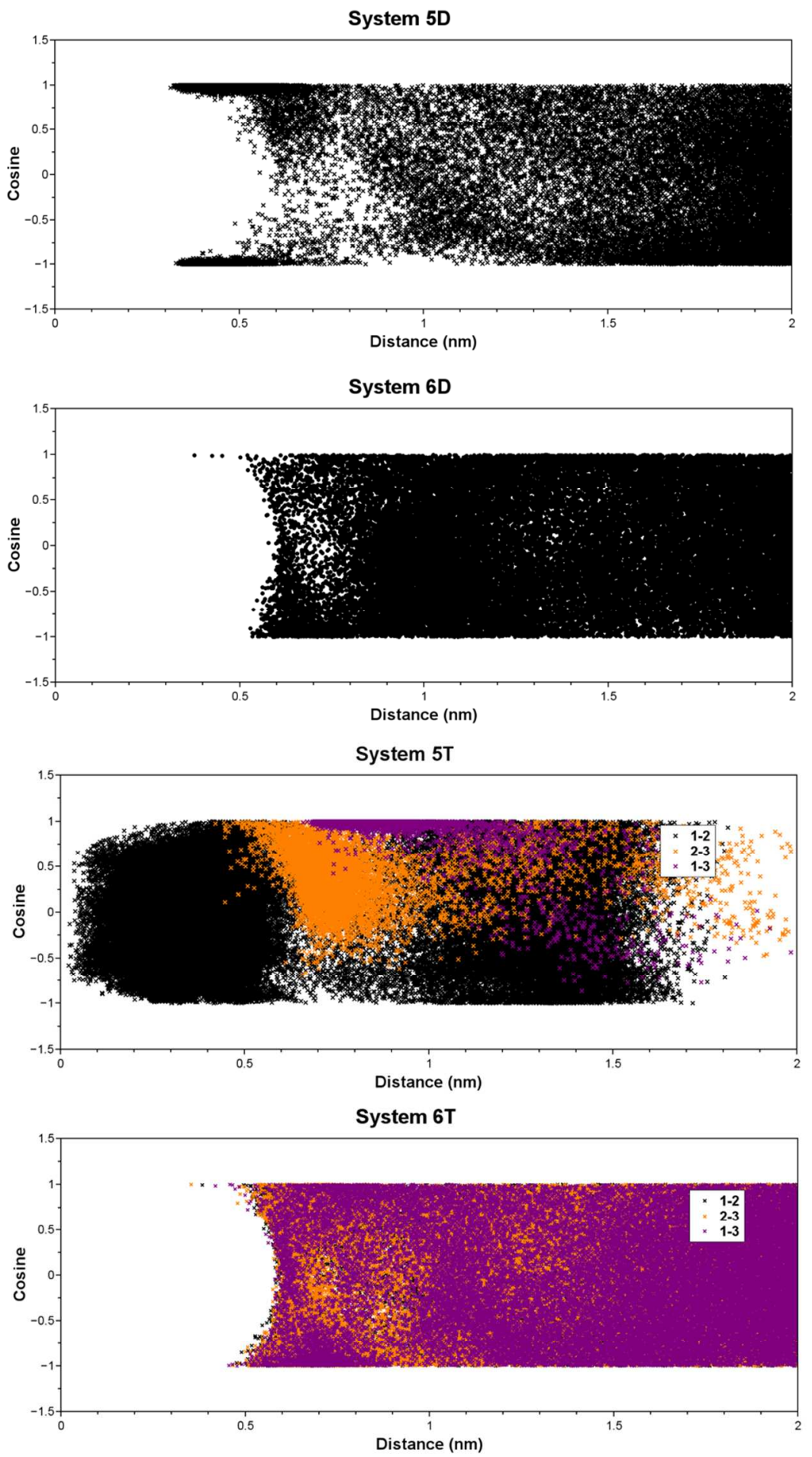

Figure S5. Cosine of angle between aromatic cores in function of distance between monomers of systems 5D, 6D, 5T, and 6T. 


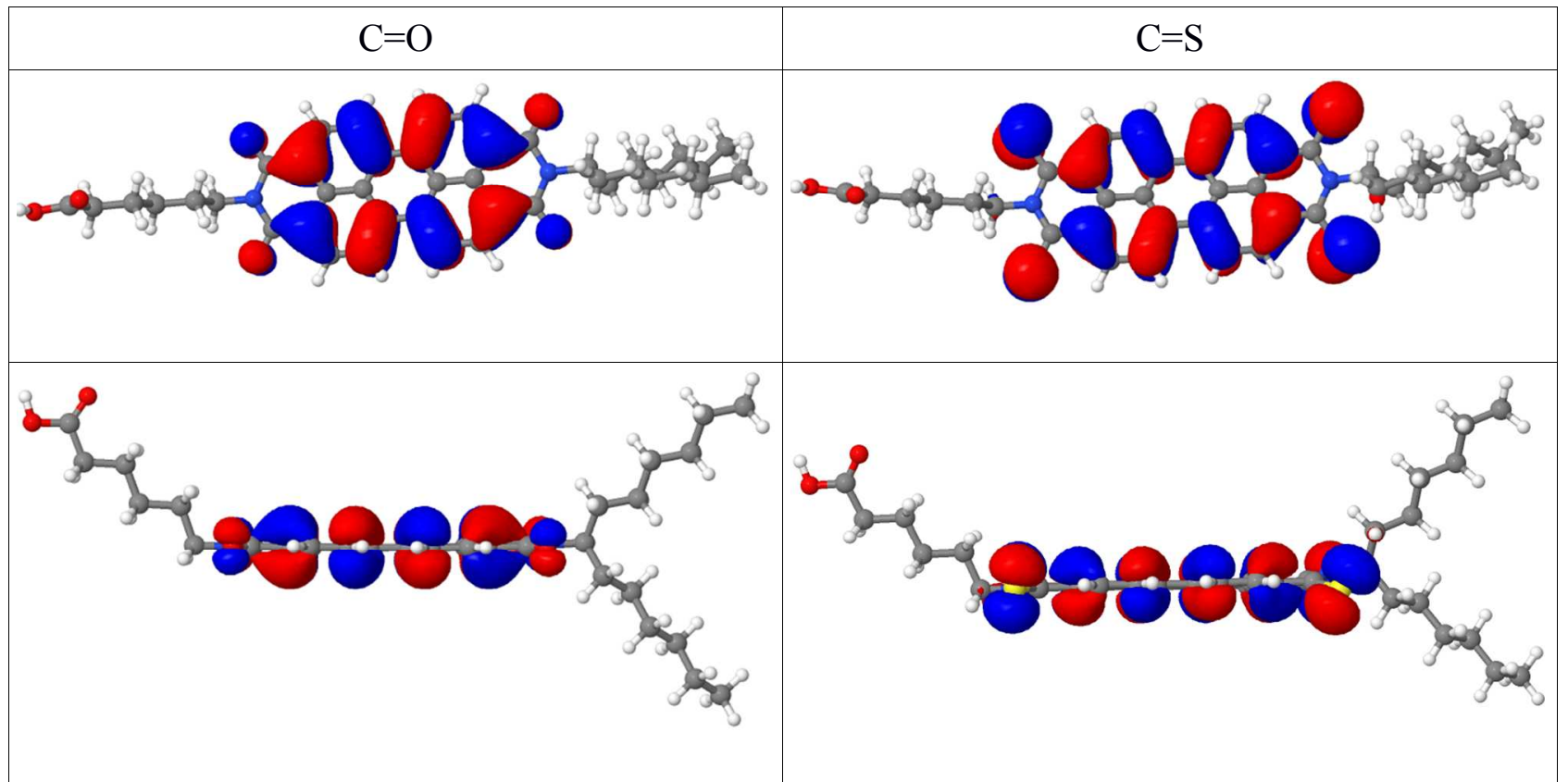

Figure S6.Top and side views of the HOMO orbitals of molecule 1 (left) and 5 (right).Carbon atoms are in grey, oxygen in red, nitrogen in blue and sulfur in yellow. 
Table S1. Systems 5D, 6D, 5T, and 6T simulated in toluene.

\begin{tabular}{cccccc}
\hline System & Compound & $\begin{array}{c}\text { Number of } \\
\text { Asphaltene } \\
\text { Molecules }\end{array}$ & $\begin{array}{c}\text { Number of } \\
\text { Solvent } \\
\text { Molecules }\end{array}$ & $\begin{array}{c}\text { Size of } \\
\text { Simulation Box } \\
\left(\mathbf{n m}^{\mathbf{3}}\right)\end{array}$ & $\begin{array}{c}\text { Simulation } \\
\text { Time (ns) }\end{array}$ \\
\hline 5D & 5 & 2 & 581 & $5 \times 5 \times 5$ & 150 \\
$6 \mathrm{D}$ & 6 & 2 & 583 & $5 \times 5 \times 5$ & 150 \\
\hline $5 \mathrm{~T}$ & 5 & 3 & 885 & $5 \times 7.5 \times 5$ & 150 \\
$6 \mathrm{~T}$ & 6 & 3 & 890 & $5 \times 7.5 \times 5$ & 150 \\
\hline
\end{tabular}

Table S2. Average distances between monomers during the molecular dynamic simulation.

\begin{tabular}{ccc}
\hline System & Average Distance 1-2 $(\mathbf{n m}) \pm$ SD & $\% *$ \\
\hline 5D & $1.45 \pm 0.86$ & 15.4 \\
6D & $1.89 \pm 0.60$ & 0.0 \\
\hline & & \\
\hline
\end{tabular}

Table S3. Average number of contacts during the molecular dynamic simulation of systems 5D, 6D, $5 \mathrm{~T}$, and $6 \mathrm{~T}$.

\begin{tabular}{ccccc}
\hline \multirow{2}{*}{ Contacts } & \multicolumn{5}{c}{ Average Number of Contacts } \\
\cline { 2 - 5 } & System 5D & System 6D & System 5T & System 6T \\
\hline$\pi-\pi$ & 165 & 14 & 252 & 48 \\
$\pi-\theta$ & 35 & 10 & 32 & 38 \\
$\theta-\theta$ & 43 & 25 & 33 & 70 \\
\hline
\end{tabular}


Table S4. Distances between monomers of trimers during the molecular dynamic simulation.

\begin{tabular}{ccccccc}
\hline System & $\begin{array}{c}\text { Average Distance 1-2 } \\
(\mathbf{n m})\end{array}$ & $\begin{array}{c}\text { \%* } \\
\text { ST }\end{array}$ & $\begin{array}{c}\text { Average Distance 2-3 } \\
(\mathbf{n m})\end{array}$ & $\mathbf{\%} *$ & $\begin{array}{c}\text { Average Distance 1-3 } \\
(\mathbf{n m})\end{array}$ & $\% * *$ \\
& $1.05 \pm 0.84$ & 5.5 & $6.01 \pm 1.83$ & 0.0 & $6.28 \pm 2.06$ & 0.5 \\
$6 \mathrm{~T}$ & $1.93 \pm 0.59$ & 0.0 & $1.76 \pm 0.64$ & 0.0 & $1.94 \pm 0.55$ & 3.8 \\
\hline
\end{tabular}

*Percentage of frames which distance is under $0.4 \mathrm{~nm}$

**Percentage of frames which distance is under $0.8 \mathrm{~nm}$ 


\section{RANDOM DISPOSITION OF MOLECULES WITHIN THE BOX}

In order to confirm that the results obtained previously are independent of the choice of starting the simulations with molecules already in an "organized", aggregated structure, we have also performed simulations starting from a random distribution of molecules within the simulation box. This was the case for molecules 1-6, dimer and trimers. The results of systems 1D-4D and 1T-4T are analyzed in the main-body of the text.

Disclaimer: these new simulations were run using absolutely the same conditions as the ones of the main body of the text or even the ones aforementioned. The only difference is that the results showed hereafter were produced using Gromacs 5.0.1 version instead of the 4.6 version. Care was taken to use absolutely the same physical and mathematical methods between the two versions.

\section{$\underline{\text { DIMERS }}$}

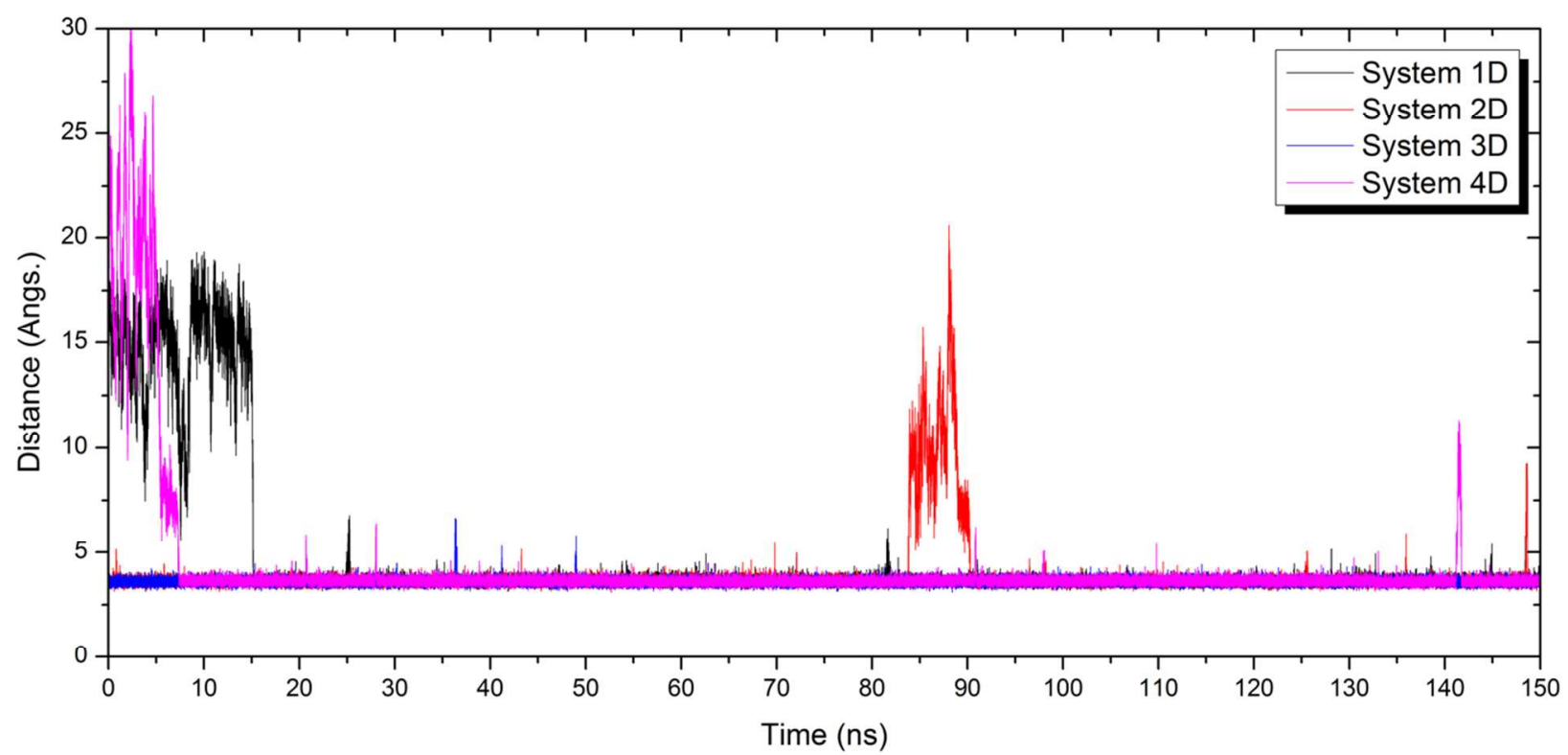

Figure S7. Minimum distances between the two molecules of the dimer systems: Molecules 1-4. 


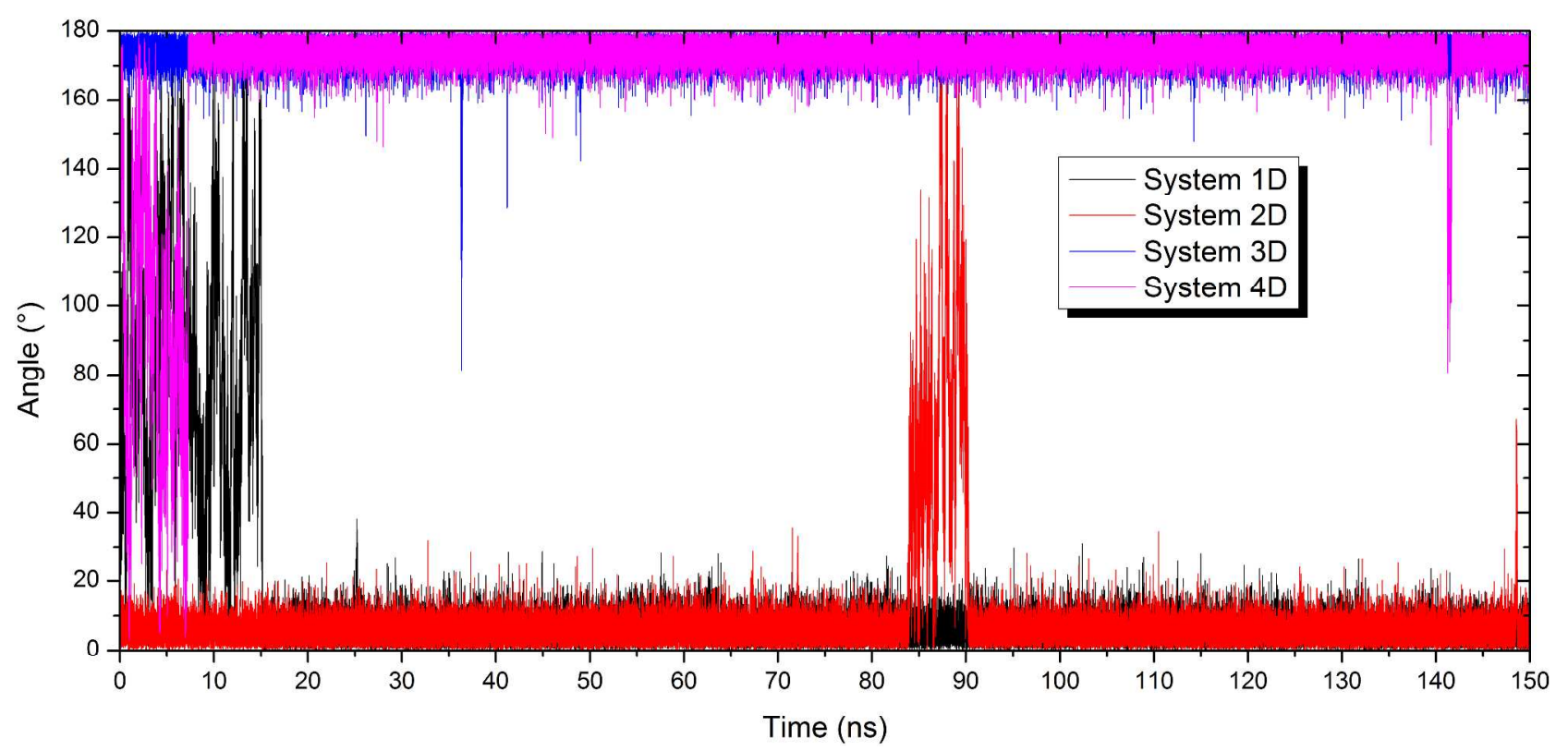

Figure S8. Angle between the two molecules of the dimer systems: Molecules 1-4.

The results obtained previously for systems 5D and 6D are now reproduced if the starting configuration is random. System 5D can assume a stable face-to-face configuration during some periods of the simulation but it is not stable throughout it. System $6 \mathrm{D}$ is not stable in this configuration and no stable conformation can be noticed by the distances analysis.

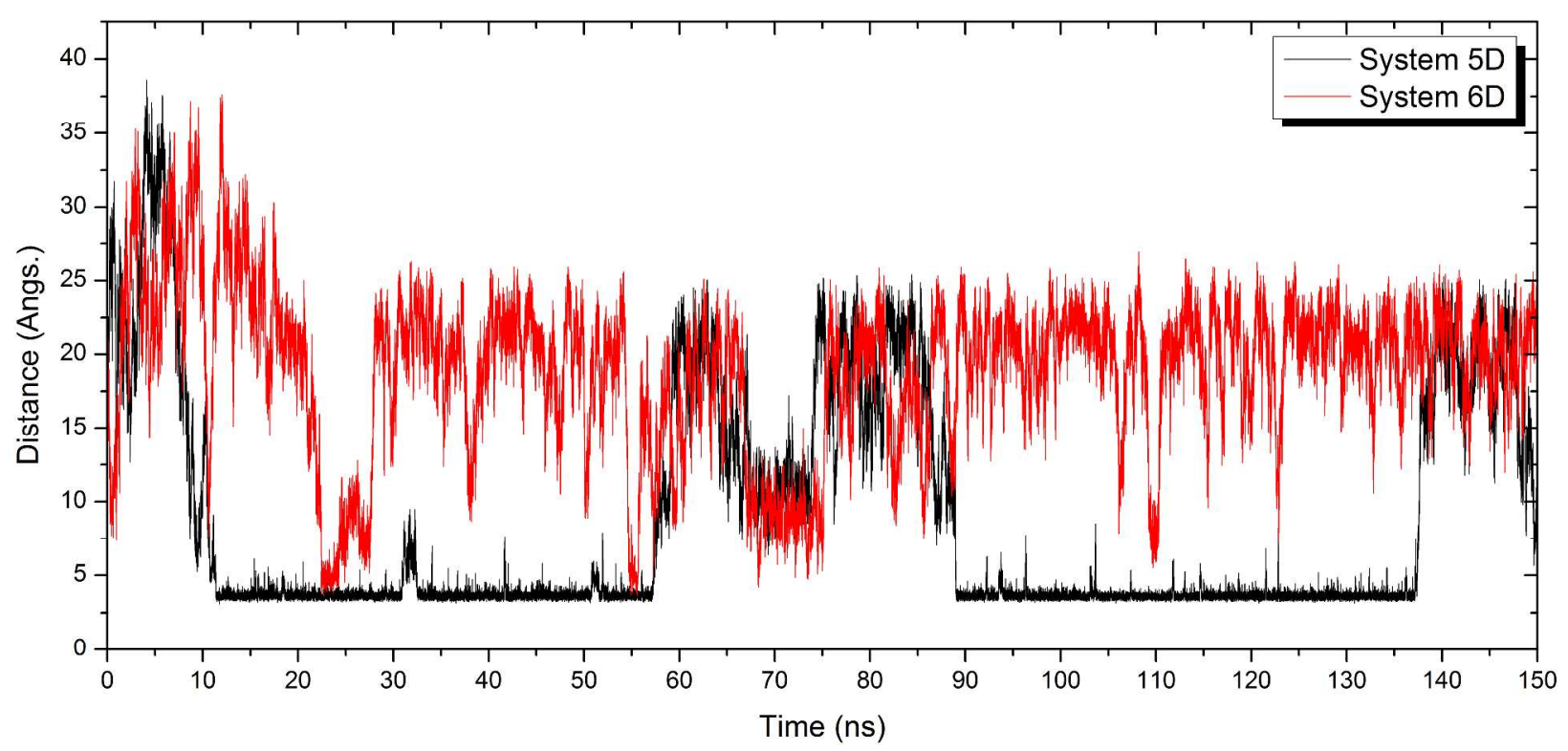

Figure S9. Minimum distances between the two molecules of the dimer systems: Molecules 5-6. 


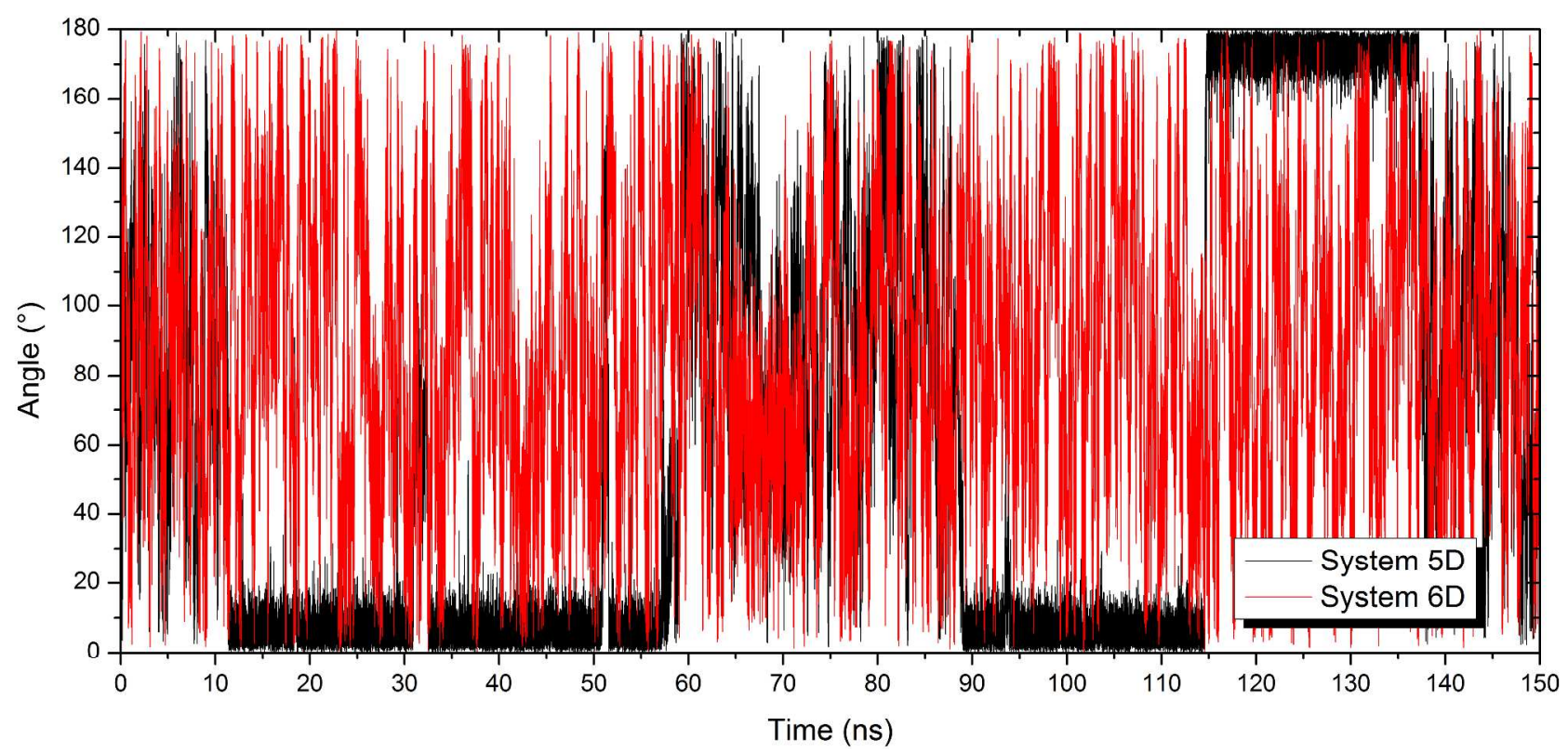

Figure S10. Angle between the two molecules of the dimer systems: Molecules 5-6. 

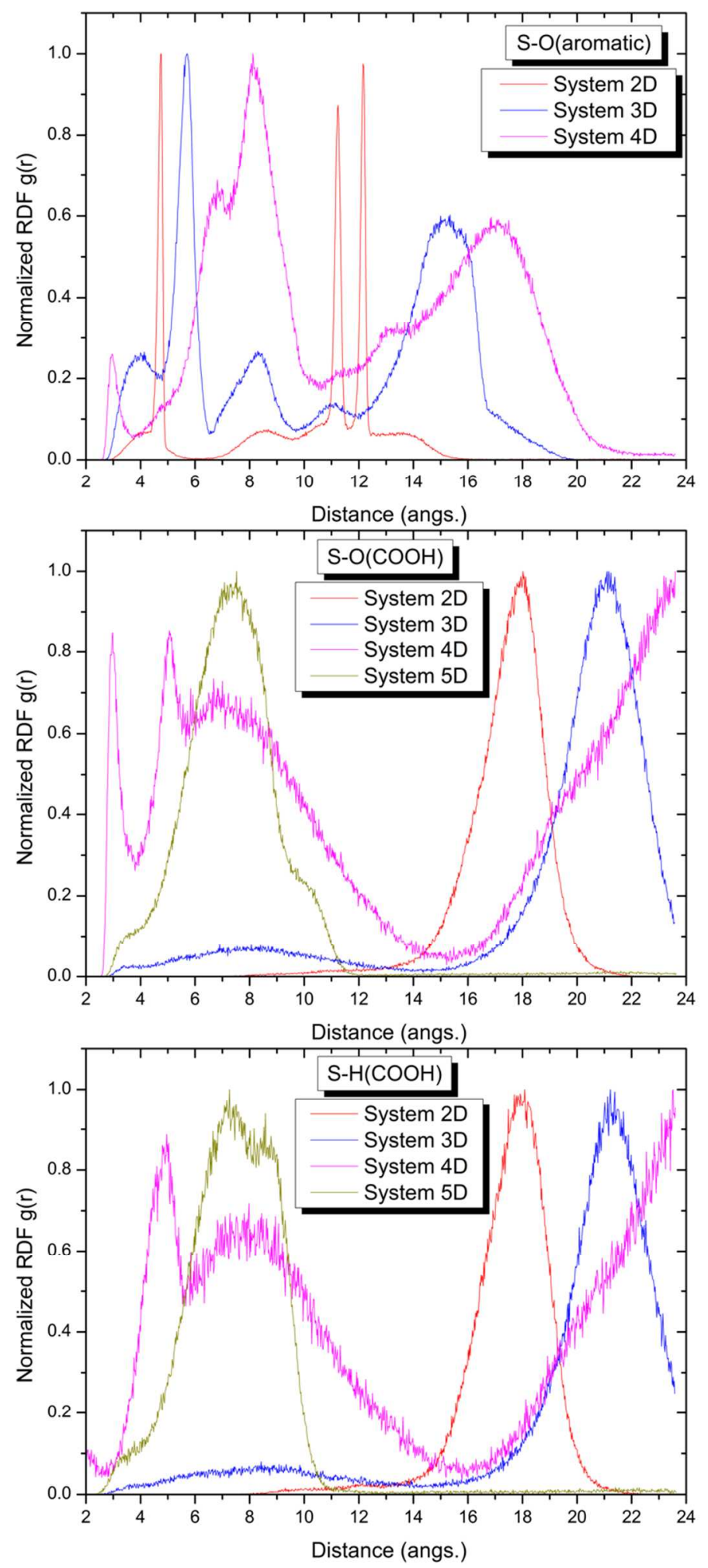

Figure S11. Normalized Radial Distribution Functions for the S-X pairwise interactions, where X can be the oxygen atom of the aromatic core, the oxygen atom of the carboxylic group $(\mathrm{COOH})$ or its hydrogen. 


\section{TRIMERS}
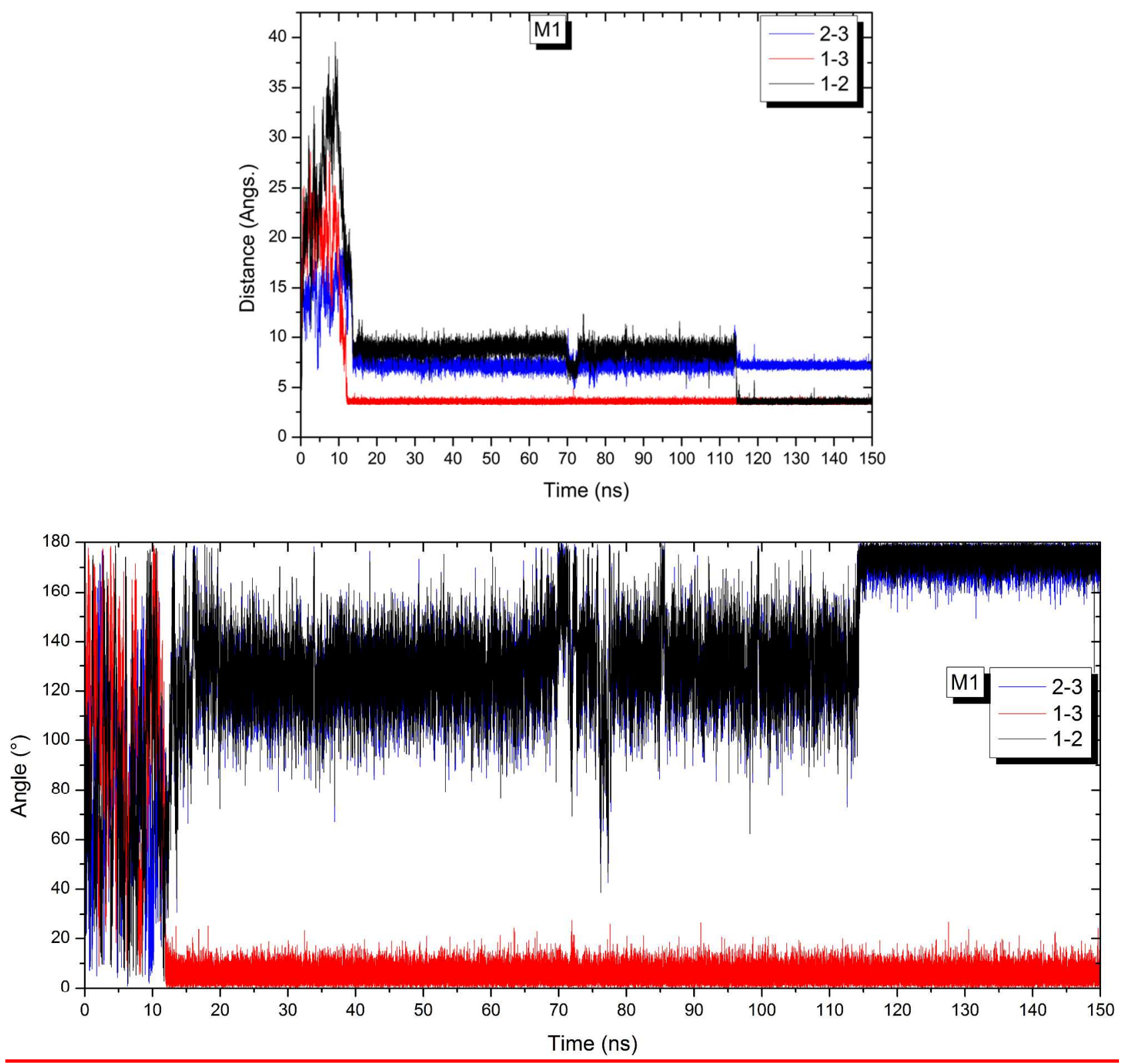

Figure S12. Minimum distances and angle between the three molecules of the trimer system 1. 

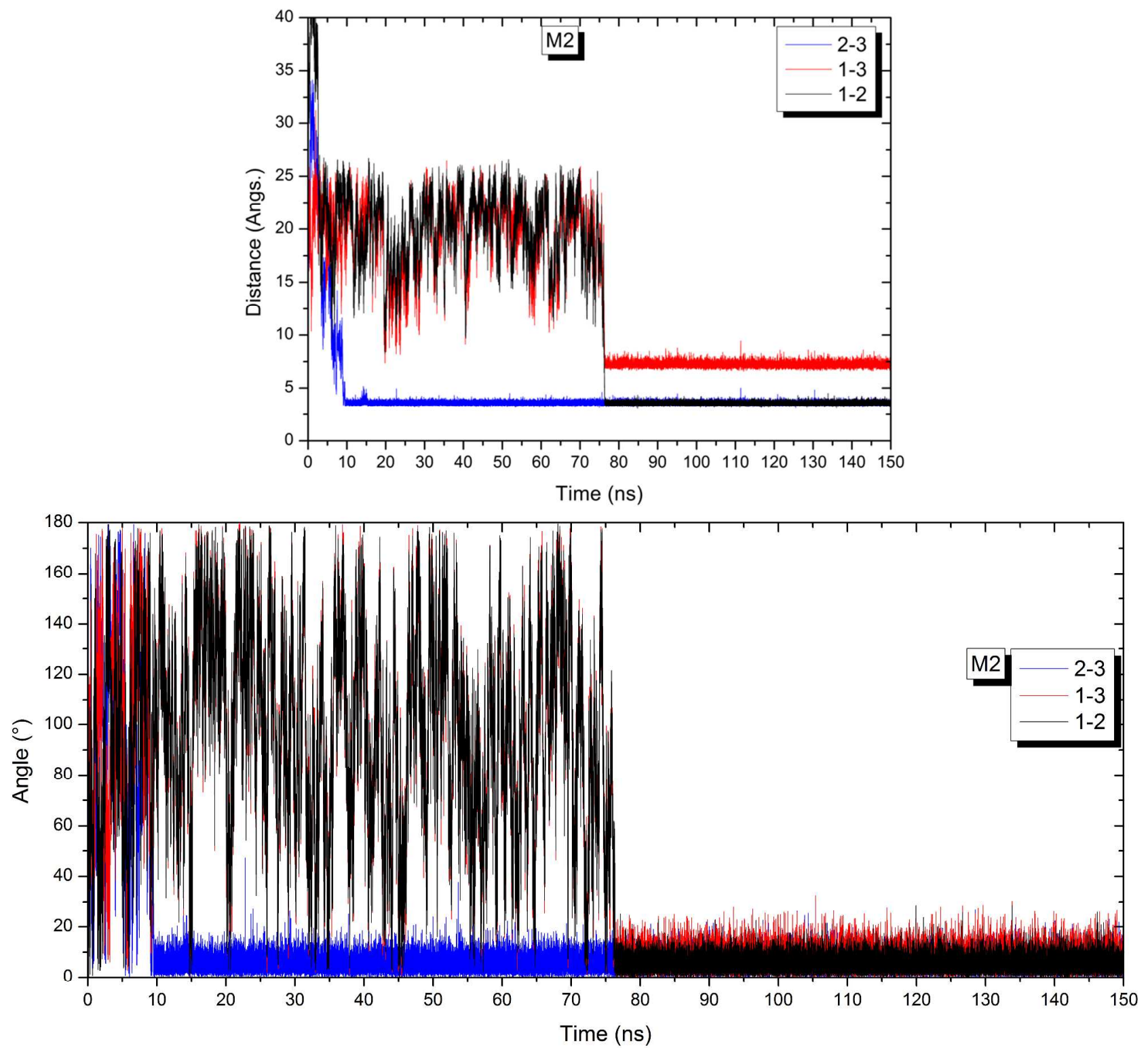

Figure S13. Minimum distances and angle between the three molecules of the trimer system 2. 

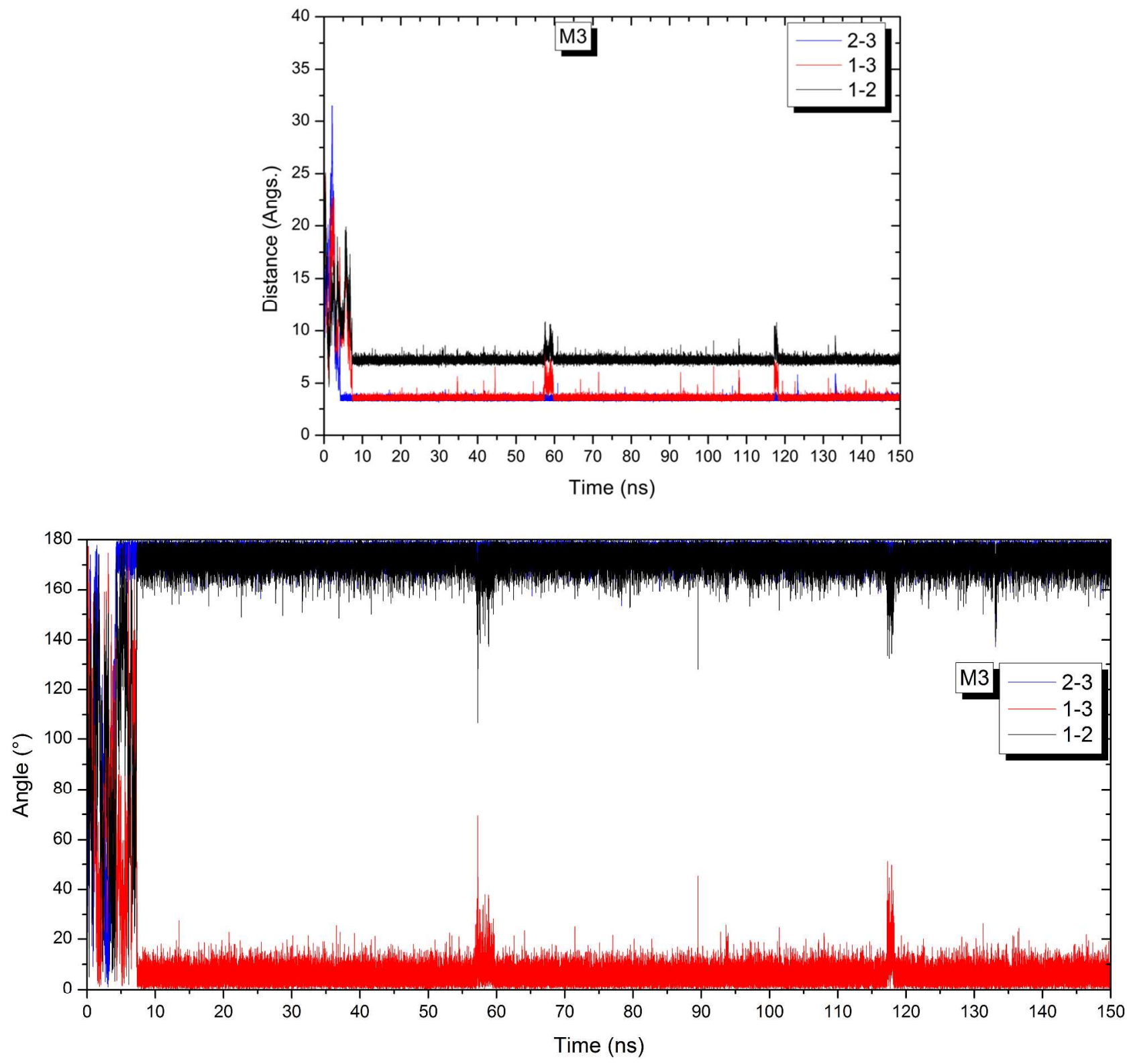

Figure S14. Minimum distances and angle between the three molecules of the trimer system 3. 

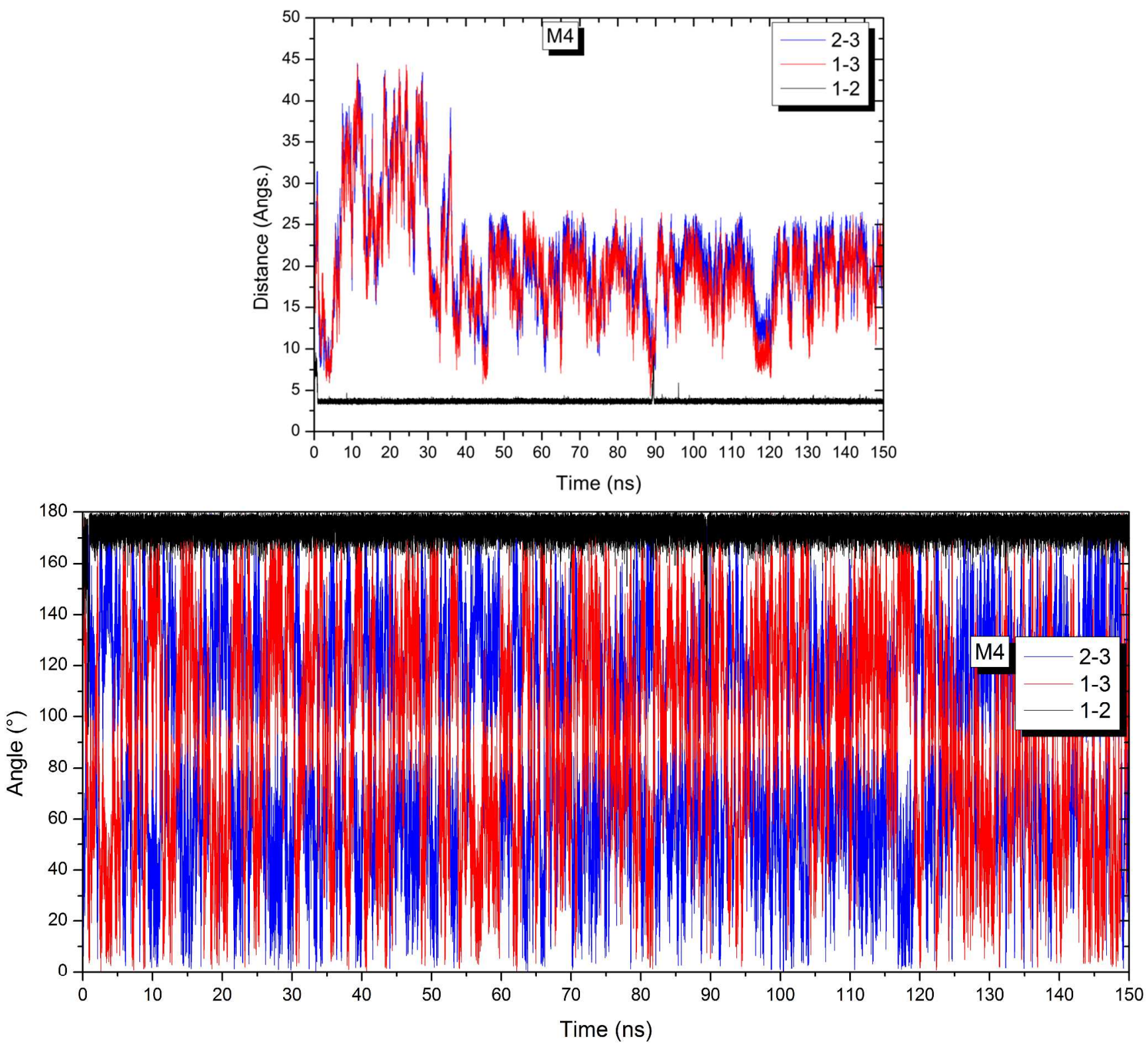

Figure S15. Minimum distances and angle between the three molecules of the trimer system 4. 

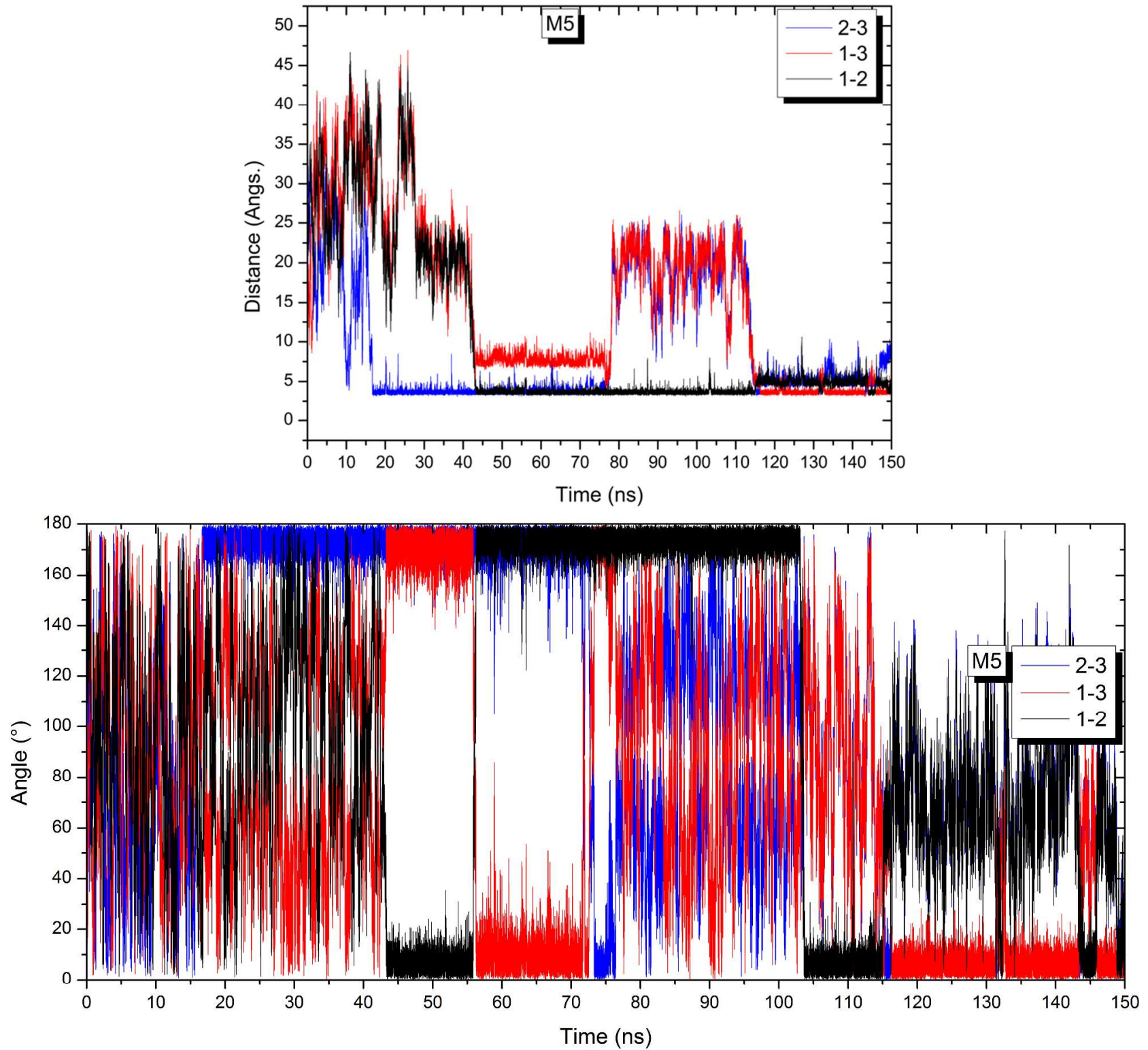

Figure S16. Minimum distances and angle between the three molecules of the trimer system 5. 

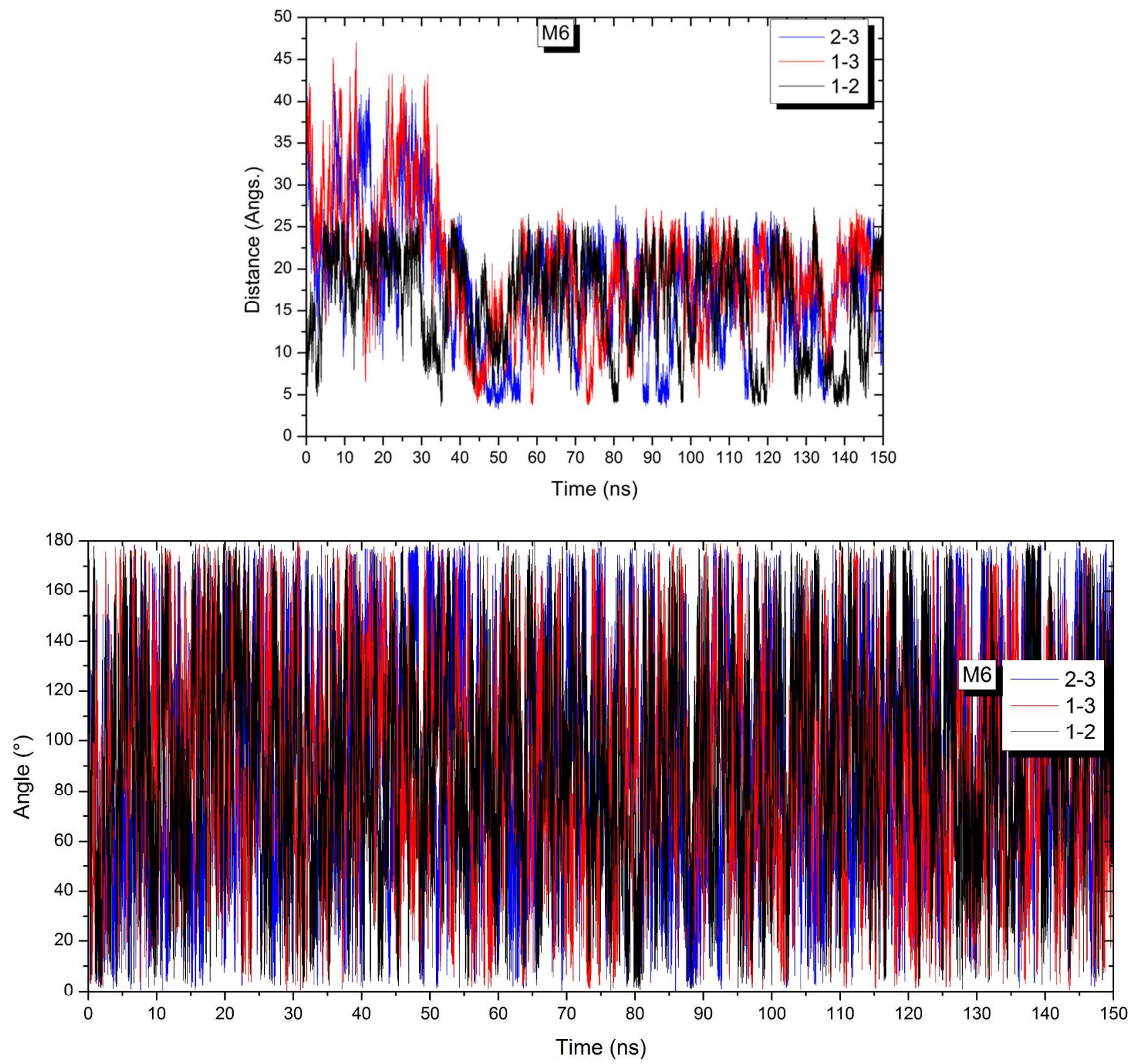

Figure S17. Minimum distances and angle between the three molecules of the trimer system 6.

These figures indicate, as previously found, that this molecule does not form stable dimers neither trimers. This might be an effect of the fact that the charge distribution of the conjugated core does not follow the same trend as for the others and this might be due to Coulomb repulsion. 

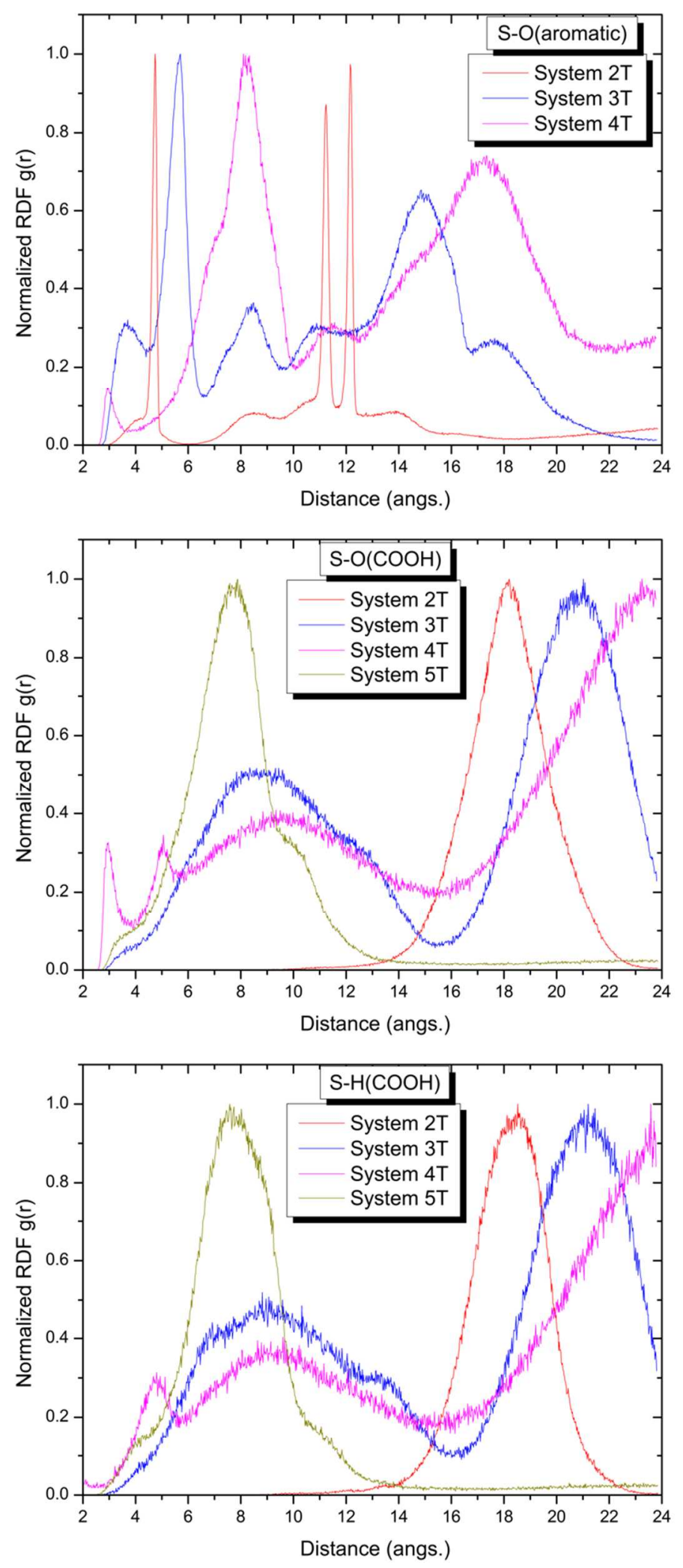

Figure S18. Normalized Radial Distribution Functions for the S-X pairwise interactions, where X can be the oxygen atom of the aromatic core, the oxygen atom of the carboxylic group $(\mathrm{COOH})$ or its hydrogen. 


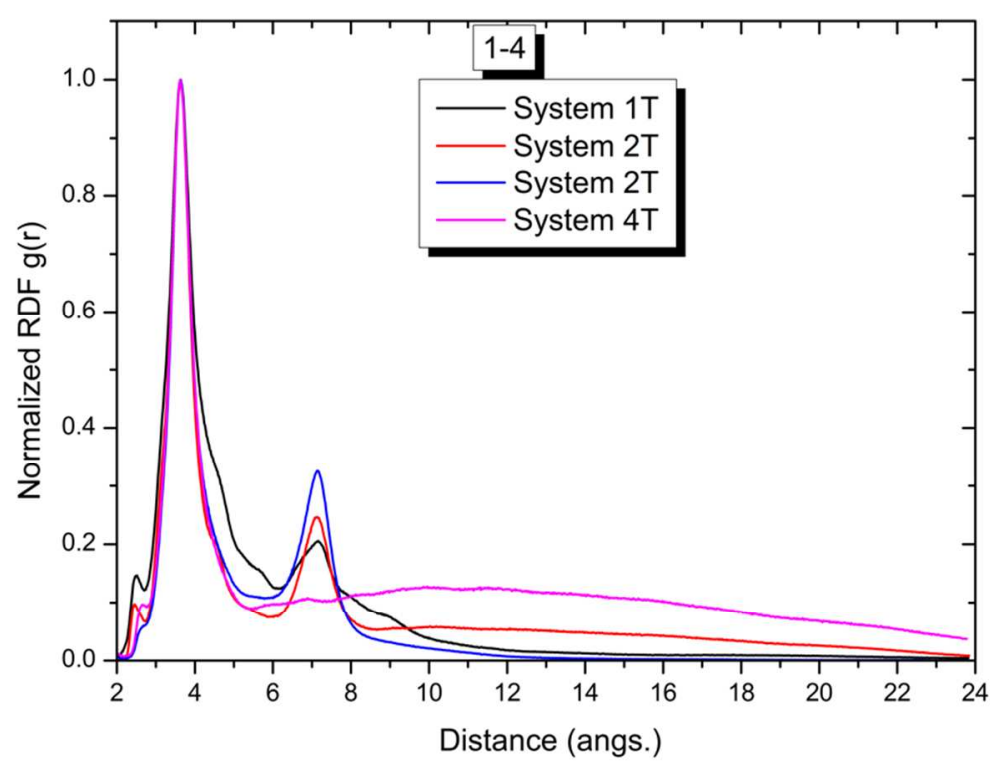

Figure S19. Normalized Radial Distribution Functions for the trimer systems containing molecules 14.

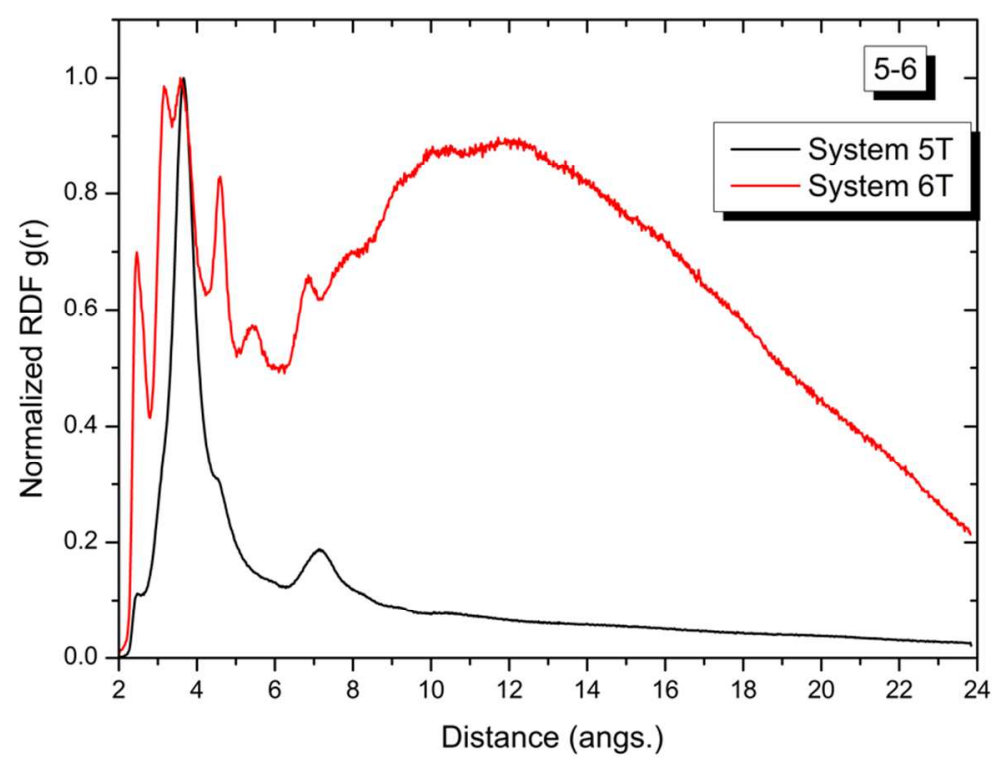

Figure S20. Normalized Radial Distribution Functions for the trimer systems containing molecules 56.

These RDF have one asphaltene molecule as reference and the other molecules as "probes". As it was showed by the analysis of the distances and angle between the planes, systems 1T-5T are relatively stable in face-to-face configuration whereas this is not the case of system 6T, very probably because the charge distribution on each monomer of this system does not follow the one for the previous molecules. Moreover, one can also note that system 4T have an aggregation pattern composed of a dimer that interacts with the other molecule through hydrogen bonding. These results were obtained for the case where the starting configuration is random. 


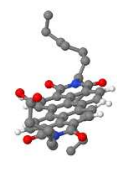

System 1D
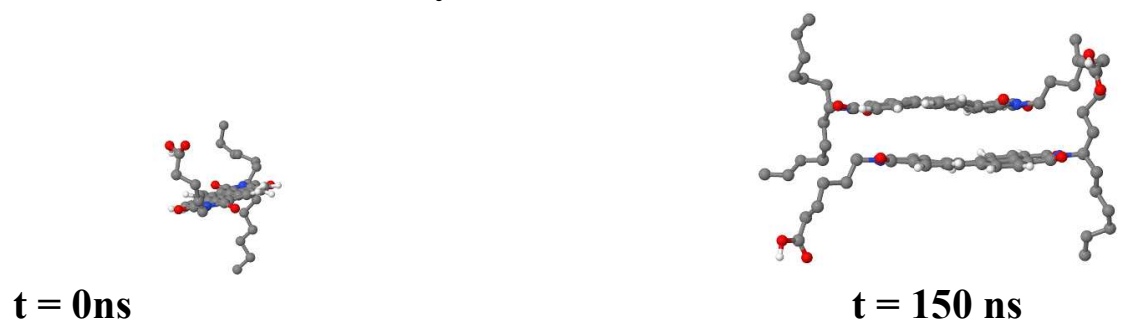

System 2D
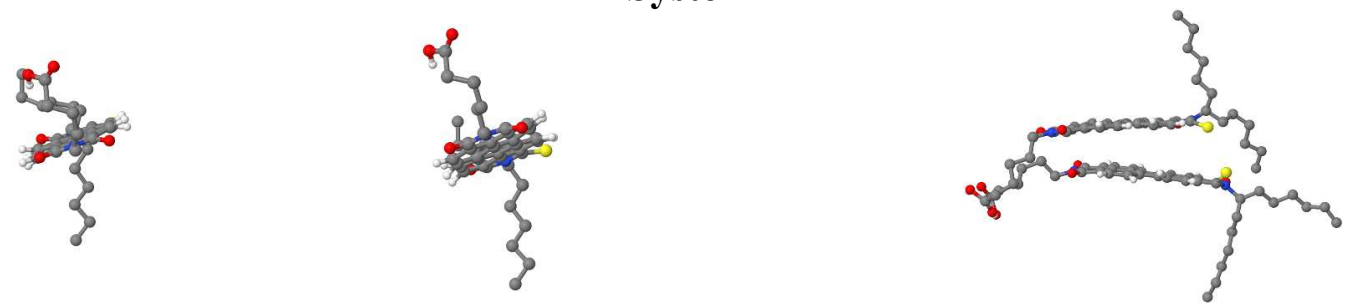

$\mathbf{t}=\mathbf{0 n s}$

$\mathrm{t}=150 \mathrm{~ns}$

System 3D
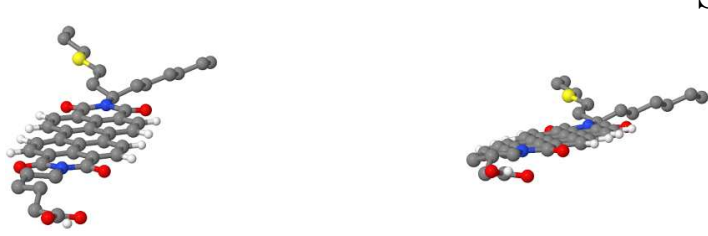

$$
\mathbf{t}=\mathbf{0 n s}
$$

System 4D
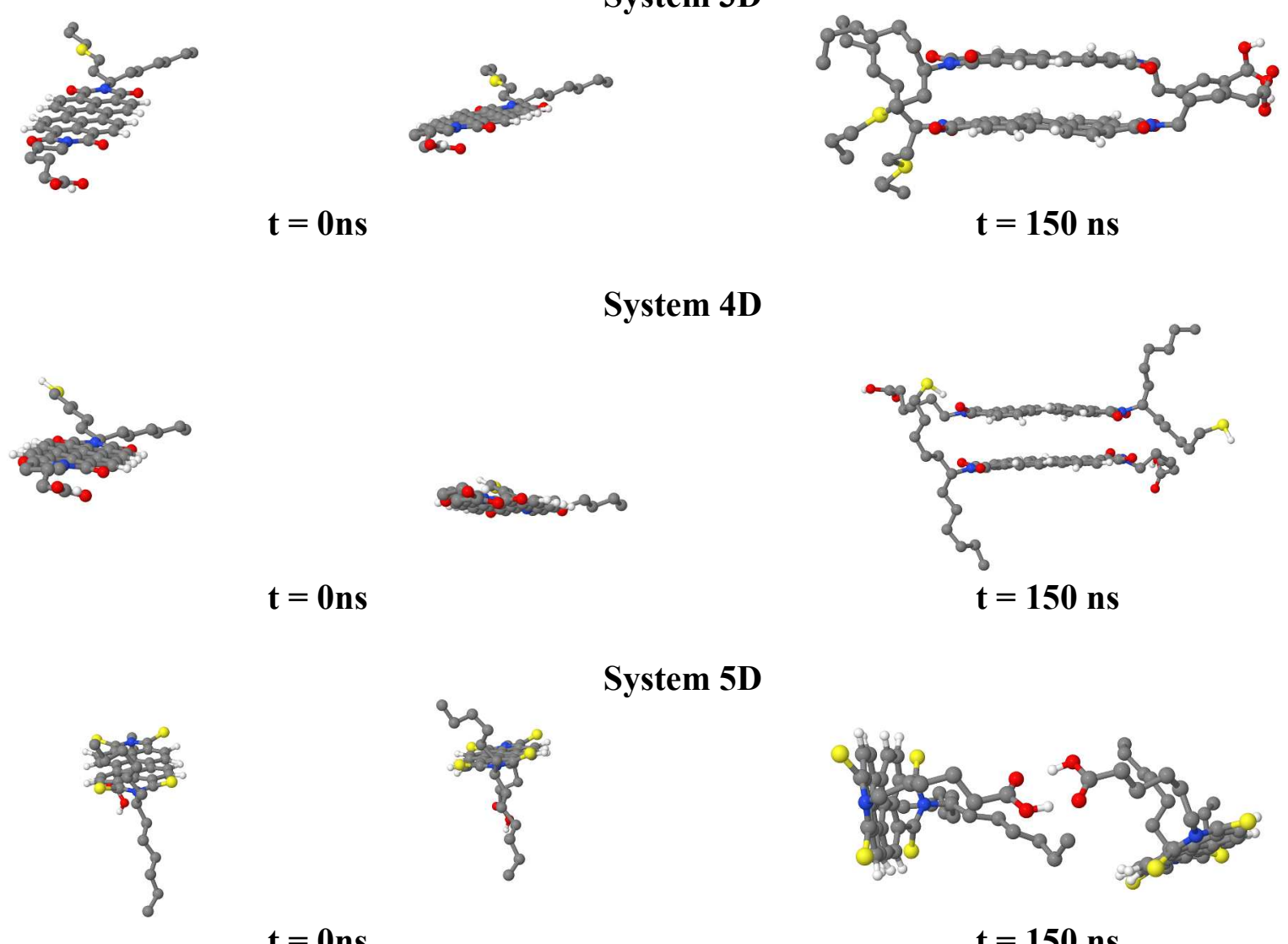

$\mathbf{t}=\mathbf{0 n s}$

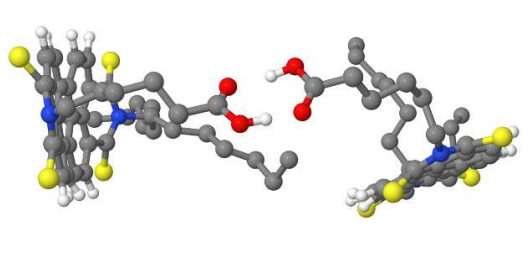

$\mathrm{t}=150 \mathrm{~ns}$ 

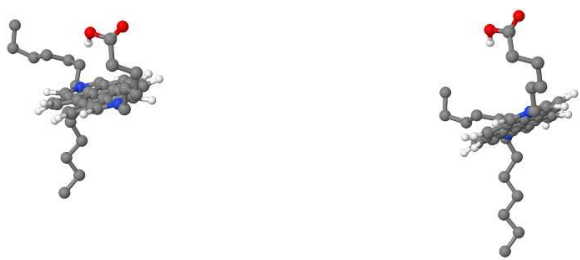

\section{System 6D}

$$
\mathbf{t}=\mathbf{0 n s}
$$

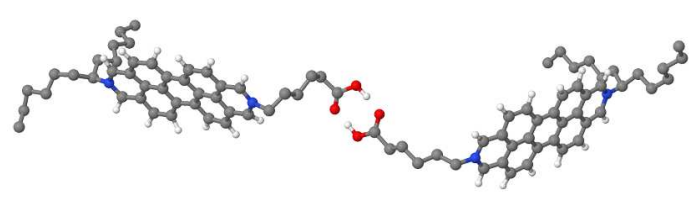

$$
\mathbf{t}=150 \mathrm{~ns}
$$

Figure S20. Snapshots of the initial configuration and the final frame of the dimers simulations for the random disposition case. Carbon atoms are in grey, oxygen in red, nitrogen in blue and sulfur in yellow. Hydrogen atoms are omitted for clarity.
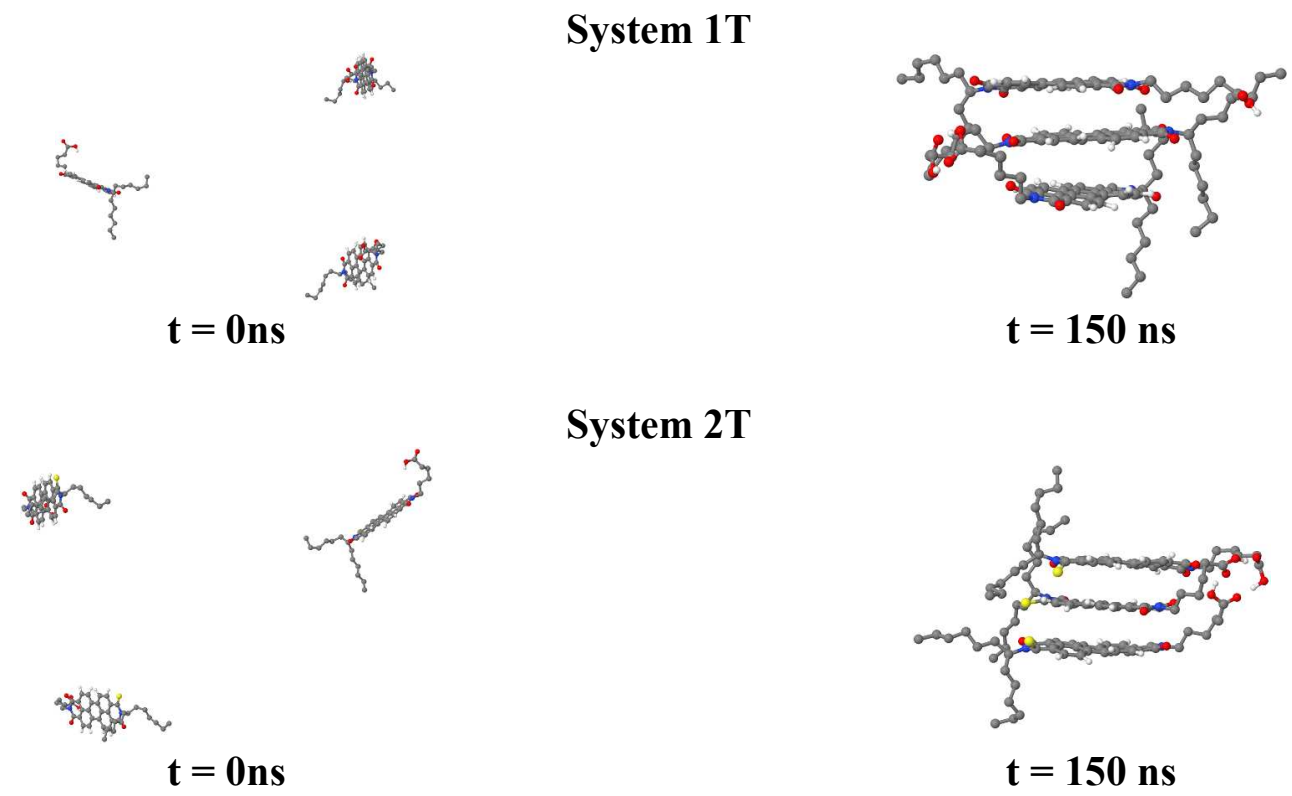

System 2T

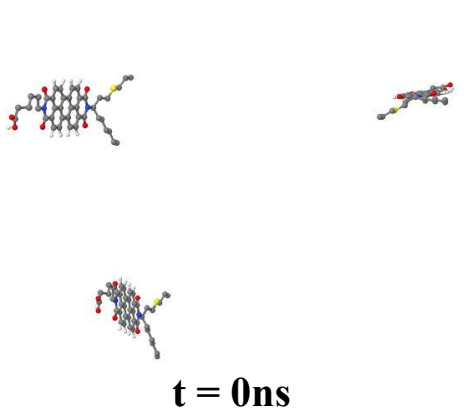

System 3T

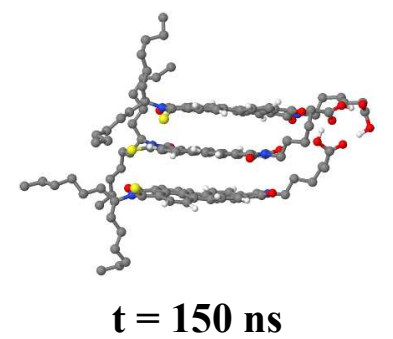

System 4T
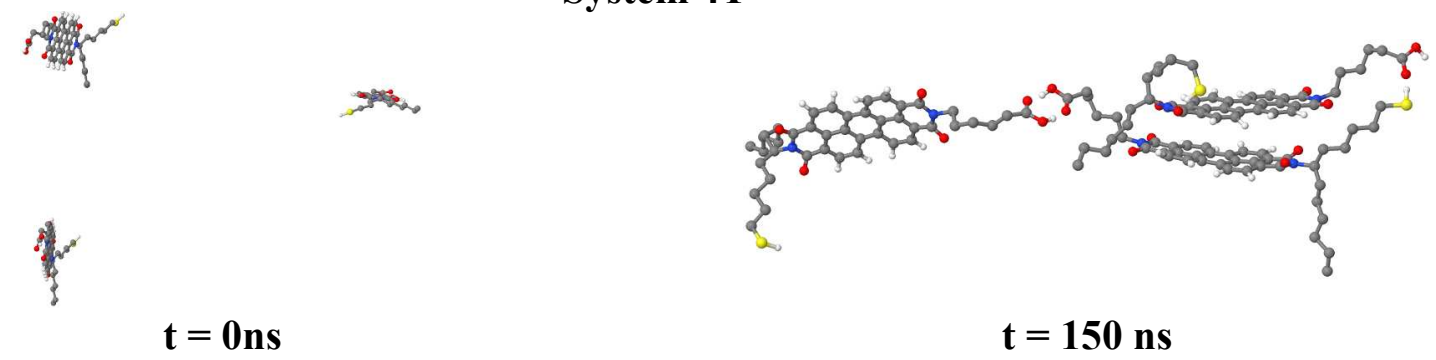

$\mathbf{t}=150 \mathrm{~ns}$

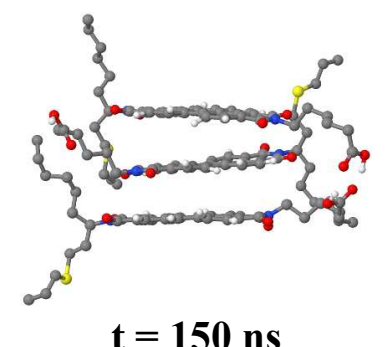



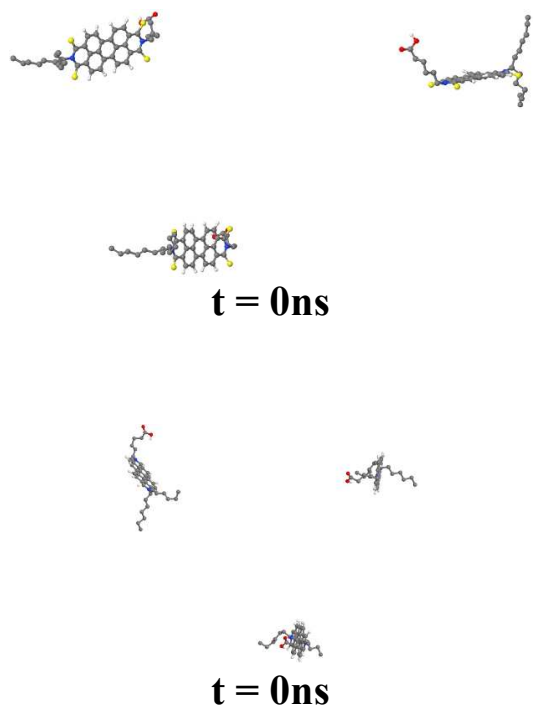

\section{System 5T}

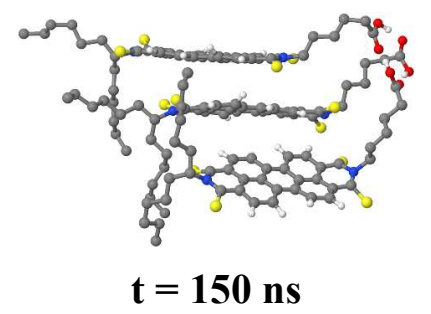

System 6T

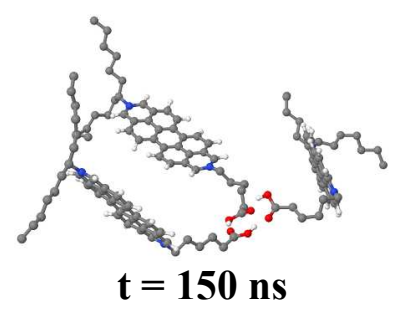

Figure S21. Snapshots of the initial configuration and the final frame of the trimers simulations for the random disposition case. Carbon atoms are in grey, oxygen in red, nitrogen in blue and sulfur in yellow. Hydrogen atoms are omitted for clarity.

\section{REFERENCES}

1. Jian, C.; Tang, T.; Bhattacharjee, S., Molecular Dynamics Investigation on the Aggregation of Violanthrone78-Based Model Asphaltenes in Toluene. Energy \& Fuels 2014, 28, (6), 3604-3613. 